\title{
„DIE DEUTSCHE IDEOLOGIE” VON MARX UND ENGELS
}

\author{
EINIGE TEXTERGÄNZUNGEN
}

In den Monaten zwischen September 1845 und dem Frühsommer 1846 schrieben Karl Marx und Friedrich Engels in Brüssel gemeinsam ihre „Kritik der neuesten deutschen Philosophie, in ihren Repräsentanten, Feuerbach, B. Bauer und Stirner" und des sogenannten "wahren" Sozialismus in Deutschland; zu diesem geplanten Buch sollte Moses Hess einige Kapitel (gegen A. Ruge und G. Kuhlmann) beisteuern. ${ }^{1}$ Die geplante Veröffentlichung unterblieb damals, weil die Geldgeber für den Druck und Verlag (J. Meyer aus Osnabrück und R. Rempel aus Bielefeld) von ihrem Versprechen zurücktraten. Die erhalten gebliebenen Teile des Manuskripts dieses Werkes von Marx und Engels das, wie Marx I8 59 schrieb, ${ }^{2}$ ihre „Selbstverständigung" zustande brachte, wurden 1932 zum ersten Mal ungekürzt publiziert. ${ }^{3}$

Die Lücken im Text waren, so weit sie nicht auf den unfertigen Charakter vor allem des Manuskripts zum Kapitel „I. Feuerbach" zurückzuführen sind, z.T. durch die "nagende Kritik der Mäuse” (Marx), z.T. aber auch dadurch hervorgerufen worden, daß mehrere Bogen nicht aufzufinden waren. Kürzlich konnten jedoch im IISG in

1 Vgl. u.a.: Marx-Engels, Briefe über „Das Kapital”, Berlin 1954, S. I4; W. Mönke, „Moses Hess und die "Deutsche Ideologie," in: Wissenschaftliche Annalen VI (r957), S. 316-330.

"Vorwort „Zur Kritik der politischen Ökonomie” (Berlin 1951, S. 14f.).

'MEGA I. Abt. Bd. 5, Berlin 1932; K. Marx, Der historische Materialismus. Hrsg. S. Landshut u. J. P. Mayer, Bd. II, Leipzig I 932 (zit. LM). Vor 1932 waren publiziert worden: Das Kapitel „Karl Grün” in O. Lünings Monatsschrift „Das Westphälische Dampfboot" III (I 847), S. 439-463, 505-525; das Vorwort zum "Leipziger Konzil" und das Kapitel „St. Bruno" von G. Mayer im "Archiv f. Soz.-wiss. u. Sozialpolitik” Bd. 47 (1920/21), S. 782-808; das Kapitel „St. Max” z.gr.T. von E. Bernstein in „Dokumente des Sozialismus" III (1903), "Arbeiter-Feuilleton" Nr. 8 (9.3.1913) und „Vorwärts" จ. I4.3.1913; die „Vorrede” und das Kapital „Feuerbach” in: Marx-EngelsArchiv (zit. MEA) I, S. 230-306. - Zur Kontroverse über die Aufbewahrung und Verwertung des Ms. vgl. u.a.: D. Rjasanow in: Protokoll. s. Kongress d. Kommunist. Internat. (r924), Bd. II, S. 940-946; ders. in MEA I, S. 205 f.; ders. in: Archiv f.d. Geschichte d. Sozialismus u.d. Arb.-bew. XI (1925), S. ${ }_{38}$, ff.; G. Mayer, ebd. XII (1926), S. 284 ff; E. Bernstein in: Der Abend (Spätausgabe des ,Vorwärts”) v. 18. 20.9.1929; Rjasanows Antwort (in 1o Expl. verteiltes Exposé) im Fried.-Adler-Archiv des IISG. 
Amsterdam, wo sich das Manuskript der "Deutschen Ideologie” - mit Ausnahme der „Vorrede” - befindet, drei Manuskriptblätter, die unter einer falschen Bezeichnung in einem mit dem Aufdruck „Drucksachen für das Mitglied des Reichstages Herrn Bernstein" und von Bernstein mit der Notiz "Der Heilige Max mit Auslassungen schon in den Dokumenten des Sozialismus gedruckt Bd III/IV" versehenen Umschlag lagen, als Teile dieses wichtigen Jugendwerkes von Marx und Engels identifiziert werden. Dieser Fund ermöglicht es, einige der erwähnten Textlücken zu schließen.

Ausserdem zeigte sich beim Vergleich zwischen Teilen des MEGATexts und des Manuskripts, das zwischen beiden gelegentlich Differenzen bestehen, die z.T. darauf zurückzuführen sind, daß der Publikation des Moskauer Instituts Fotokopien zu Grunde lagen, so daß bei Faltungen im Papier Textverluste entstanden oder von Eduard Bernstein vorgenommene Änderungen (vor allem Streichungen) nicht von solchen der Verfasser zu unterscheiden waren. ${ }^{1}$

Bei den als Teile der „Deutschen Ideologie” identifizierten Blättern handelt es sich um von Engels geschriebene, von Marx korrigierte Manuskripte, die in Format, Papier etc. den bisher bekannten Manuskriptblättern entsprechen. Auf dem ersten Blatt stand ursprünglich ein von Marx korrigierter, dann durchgestrichener Text, der weitgehend mit den im Kapitel „II. Sankt Bruno” der MEGA auf den Seiten 84 (Zeile 17-21, 35-37), 85 (Z. I I-37) und 86 (Z. 1-29) abgedruckten Ausführungen übereinstimmt. ${ }^{2}$ Später hat Engels in die rechte Spalte der Vorderseite einen Teil von "I. Feuerbach" geschrieben, der sich als die Fortsetzung des Texts in der MEGA, I. Abt., Bd. 5, S. 32, Zeile s identifizieren ließ; Marx hat diese Seite neu mit der Seitenzahl 29 versehen. Das zweite Blatt ist deswegen besonders interressant, weil Marx die rechte Spalte der Vorderseite völlig mit Zeichnungen, Randbemerkungen und Schreibübungen ausgefüllt hat (s. Bildtafel). Nach mehreren später wieder ausgestri-

${ }^{1}$ Einige Beispiele: MEGA 1/5, S. 64, Z. I-2 nach „Gemeinschaft” „existiren für jedes Individuum” (bei LM S. 60 „erhält das Individuum”, dto. MEA I, S. 286); MEGA I/5 S. 590 , Anm. I : von Bernstein gestrichen, also „als unkommunistisch” auf S. I 89 Z. 14 einzufügen und ebd. Z. I6 nach "99." einzufügen „Es finden sich..." (S. 590 unten); ebd. S. 591 zu S. 197 Z. 39 vor „kämpfen” "[Ge]sindel zu”; zu S. I95 Z. 16 nach „Proudhon" die Fußnote (S. 591) einzuschieben bis einschl. "scharf kritısierte”; S. 205 Z. 6 von S. $592 \mathrm{ab}$ "In Frankreich" einzufügen (dies meist richtig in LM). Ferner sind z.B. in den Haupttext einzufügen: Auf S. 21 Z. 20 nach „einstellt" von S. 571 ,wo dieser Nation” bis incl. "diese nationale" (Rest des Satzes im Ms. getilgt; richtig LM S. 22 u. MEA I, S. 249); auf S. 25 Z. 38 hinter ,ist" von S. 573 von "bisher" bis "Gesellschaft" (richtig LM S. 28 ; MEA I, S. 254). Diese Stellen fehlen in den populären Ausgaben völlig; jedoch wird die zuletzt genannte Stelle in Marx-Engels, Werke (zit. MEW), Bd. 3, Berlin 1959, in einer Anm. auf S. 36 mit dem falschen Zusatz „Im Ms. gestrichen” erwähnt.

${ }^{2}$ Anhang, S. ror $f$. 
chenen Sätzen ${ }^{1}$ wird auf dieser Seite und dem oberen Teil der Rückseite der von Engels geschriebene Text des ersten Blatts fortgesetzt. Auf der durch einen Trennungsstrich von der oberen geschiedenen unteren Hälfte der Rückseite findet sich die „Urfassung” des in der MEGA I/s auf Seite 75, Zeile 22 bis Seite 76, Zeile 6 abgedruckten Absatzes aus dem Beginn des Kapitels „II. Sankt Bruno”.2 Auf diesem Blatt finden sich sehr viele Korrekturen, Randbemerkungen und Streichungen von Marx, der auch die Seiten mit den Ziffern I und 2 gekennzeichnet hat. Das dritte Blatt schliesslich, von Engels auf der Vorderseite mit der Ziffer 28 versehen, enthielt - wieder von Engels geschrieben ${ }^{3}$ - den Anfang des die Lücke im Kapitel „III. Sankt Max", MEGA I/s, Seite I80, Zeile 20 ausfüllenden Textes; anscheinend fehlt hier weiterhin eine Fortsetzung als Úberleitung zum Beginn des bisher bekannten Textes (ebd. S. 180, Z. 22). Auf diesem Blatt finden sich von Marx nur eine Bleistiftkorrektur unten auf dem rechten Rand der Vorderseite und die Seitenangabe auf der Rückseite. - Das zweite Blatt ist unten, das dritte Blatt oben stark beschädigt.

Bei der Wiedergabe der Texte und - im Anhang - der Textvarianten wurde aus praktischen Gründen die Textgestaltung der MEGA zum Vorbild genommen, - allerdings ohne Modernisierung des Textes. ${ }^{4}$

1 Anhang, S. 102, Z. 35.

Anhang, S. IO3 (Mitte).

- Schon G. Mayer stellte mit Recht fest: „In der Tat ist das Manuskript fast ganz von Engels geschrieben ... Allein für dic Verfasserschaft besagt dieser handschriftliche Tatbestand nicht das mindeste. ... Engels schrieb leserlicher, er war quicker und fixer, und deshalb stets bereit, Abschnitte, die er mit Marx gemeinsam entworfen hatte, aufs Papier zu bringen." (Arch. f. Soz.-Wiss. a.a.O., S. 776).

"Zur Erleichterung der Einordnung für den Leser wird auch auf die entsprechenden Seiten der MEW und LM hingewiesen. 
(I Einzufügen im Kapitel „I. Feuerbach”, MEGA I/s, S. 32, Z. s; LM, Bd. II, S. 37; MEW, Bd. 3, S. 42 (hinter „ihrem”).

|29/ „Wesen” nicht im Entferntesten entspricht, so wäre dies nach der erwähnten Stelle ein unvermeidliches Unglück, das man ruhig ertragen müsse. Diese Millionen Proletarier oder Kommunisten, denken indeß ganz anders, und werden dies ihrer Zeit beweisen, wenn sie ihr ,Sein" mit ihrem "Wesen" praktisch, durch eine Revolution in Einklang bringen werden. Bei solchen Fällen spricht Feuerbach ${ }^{1}$ daher nie von der Menschenwelt, sondern er flüchtet sich jedesmal in die äußere Natur, und zwar in die Natur, die noch nicht unter die 10 Herrschaft der Menschen gebracht ist. Mit jeder neuen Erfindung aber, mit jedem Fortschritt der Industrie wird von diesem Terrain ein neues Stück abgerissen, und der Boden, auf dem die Beispiele für ähnliche Feuerbachsche Sätze wachsen, wird so immer kleiner. Das „Wesen” des Fisches ist sein „Sein”, das Wasser, um bei dem einen Satze stehen zu bleiben. Das „Wesen” des Flußfisches ist das Wasser eines Flusses. Aber dies hört auf, sein „Wesen" zu sein, es wird ein für ihn nicht mehr passendes Existenzmedium, sobald dieser Fluß der Industrie unterthan gemacht, sobald er durch Farbstoffe und sonstige Abfälle verunreinigt, durch Dampfschiffe befahren, sobald 20 sein Wasser in Gräben geleitet wird in denen man dem Fisch sein Existenzmedium durch einfaches Ablassen entziehen kann. Diese Erklärung aller derartigen Widersprüche zu einer unvermeidlichen Abnormität ist im Grunde von dem Trost nicht verschieden, den der heilige Max Stirner ${ }^{2}$ den Unzufriedenen gibt, daß nämlich dieser Widerspruch ihr eigner Widerspruch diese schlechte Lage ihre eigne schlechte Lage sei, wobei sie sich entweder beruhigen könnten, oder ihren eignen Widerwillen für sich behalten, oder sich auf phantastische Weise dagegen empören dürften - und ebenso wenig verschieden von dem Vorwurfe des heiligen Bruno, ${ }^{3}$ dass diese un- ${ }^{3}$ glückseligen Umstände daher kämen, daß die Betreffenden im Dreck der „Substanz” stecken geblieben, nicht zum ,absoluten Selbstbewußtsein" fortgeschritten seien und diese schlechten Verhältnisse nicht als Geist von ihrem Geist erkannt hätten.

/I/ Wir werden uns natürlich nicht die Mühe geben ${ }^{4}$, unsere weisen

${ }^{1}$ Ludwig Feuerbach, Grundsätze der Philosophie der Zukunft (1843). In: Sämmtliche Werke, Bd. II, Stuttgart 1904, S. $247 \mathrm{ff}$, bes. S. 286.

2 Vgl. Max Stirner, Der Einzige und sein Eigenthum, Leipzig 1845, S. I 55 f., I 7 I f.

3 Bruno Bauer, "Charakteristik Ludwig Feuerbachs". In: Wigand's Vierteljahrsschrift, Jg. I 845 , Bd. 3, S. 86-146, bes. S. 87, I09ff., I 38 f. Vgl. Norddeutsche Blätter, Jg. I 844, Heft IV, S. I-13.

4 Marx in der rechten Spalte: Feuerbach. 
Philosophen darüber aufzuklären 1, daß die „Befreiung” des „Menschen" damit noch um keinen Schritt weiter gekommen ist ${ }^{2}$, wenn sie Philosophie, Theologie, Substanz und den ganzen Unrath in das „Selbstbewußtsein" aufgelöst ${ }^{3}$, wenn sie den Menschen von der

5 Herrschaft dieser Phrasen 4, unter der er nie geknechtet war, befreit haben ${ }^{5}$; daß es nicht möglich ist, eine wirkliche Befreiung anders als in der wirklichen Welt und mit wirklichen Mitteln durchzusetzen, daß man die Sklaverei nicht aufheben kann ohne die Dampfmaschine und die Mule-Jenny, die Leibeigenschaft nicht ohne verbesserten Ackerbau, daß man überhaupt die Menschen nicht befreien kann, solange sie nicht im Stande sind, sich Essen und Trinken, Wohnung und Kleidung in vollständiger Qualität und Quantität zu verschaffen. Die "Befreiung” ist eine geschichtliche That, keine Gedankenthat, und sie wird bewirkt durch geschichtliche Verhältnisse, durch den [Sta]nd der Industrie, des Han[del]s, [des Acker]baus, der Ver[kehrsverhältnisse $][\ldots]^{6}$

/2/ dann nachträglich, je nach ihren verschiednen Entwicklungsstufen, den Unsinn von Substanz, Subjekt, Selbstbewußtsein und reiner Kritik gerade wie den religiösen und theologischen Unsinn, und beseitigen ihn nachher wieder ${ }^{7}$, wenn sie weitgenug entwickelt sind. Natürlich ersetzen in einem Lande wie Deutschland ${ }^{8}$, wo nur eine lumpige geschichtliche Entwicklung vor sich geht, diese $\mathrm{Ge}-$ dankenentwicklungen, diese verklärten und thatlosen Lumpereien den Mangel der geschichtlichen ein ${ }^{9}$, und setzen sich fest und müssen bekämpft werden. Aber das ist ein Kampf von lokaler Bedeutung . ${ }^{10}$

1 Zu den Randbemerkungen von Marx auf dieser Höhe vgl. S. ro2, Anm. I.

- Marx in der rechten Spalte: Philosophische, u. wirkliche Befreiung.

3 Id.: Der Mensch. Der Einzige. Das Individurum.

Id.: Geologische, hydrographische etc. Bedingungen

- Id.: Der menschliche Körper. D. Bedürfniß u. d. Arbeit

- [ ] im Haupttext = Blatt beschädigt.

7 Marx in der rechten Spalte: Phrasen u. wirkliche Bewegung.

- Id.: Bedeutung der Phrasen für Deutschld.

- Versehentlich nicht ausgestrichen von der früheren Konstruktion: nehmen... die Stelle... ein.

${ }^{10}$ Marx in der rechten Spalte: D. Sprache ist d. Sprache d. Wi[rklichkeit] - Es folgt nach einem Trennungsstrich ein getilgter Absatz, der fast wörtlich mit den Ausführungen MEGA I/5, S. 75, Z. 22 bis S. 76, Z. 6 übereinstimmt (s. Anhang). 


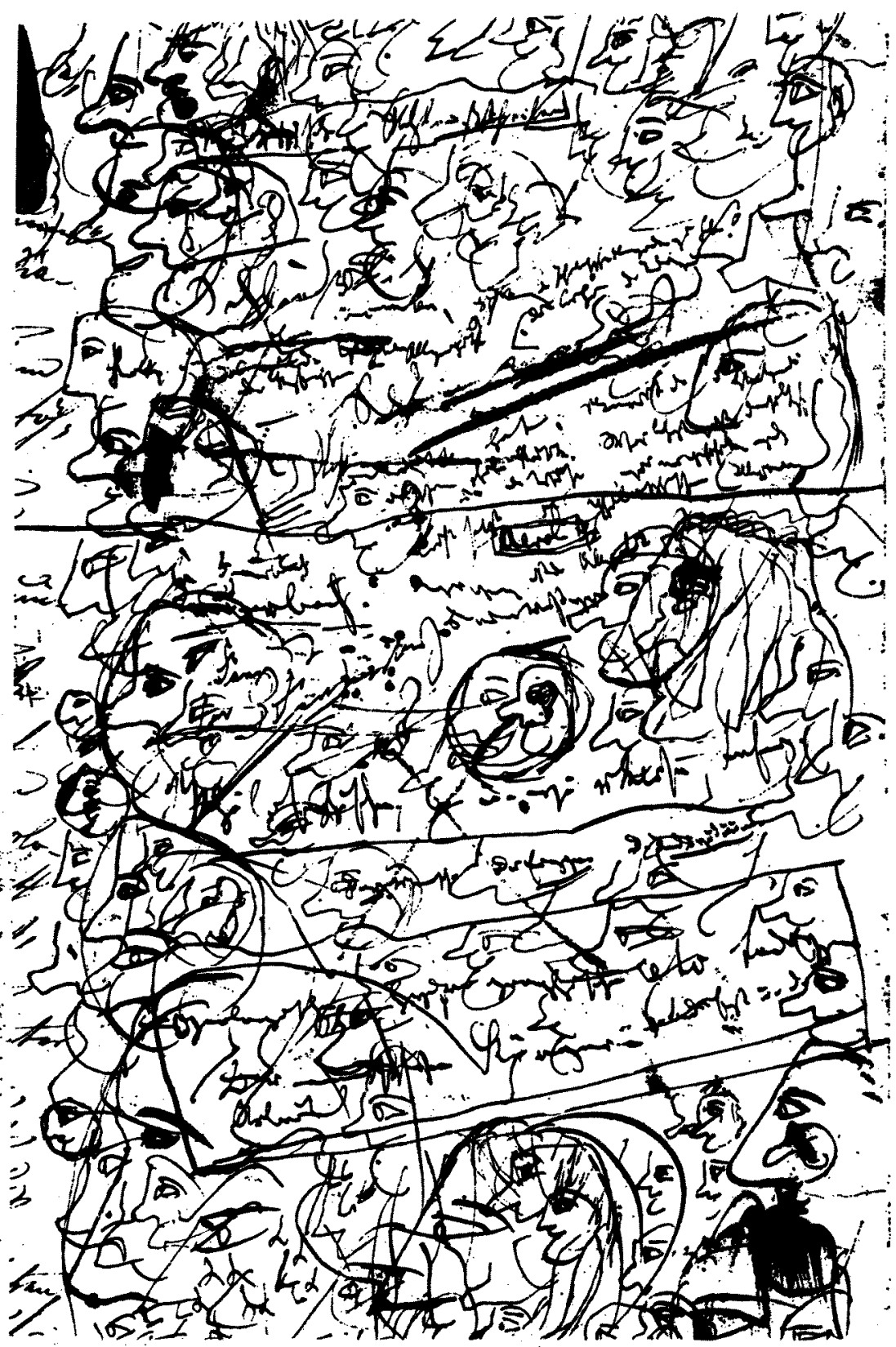

Stück der S. I des Manuskripts mit einem Teil der Zeichnungen und den Randbemerkungen von Marx. 
II) Einzufügen im Kapitel „III. Sankt Max”, MEGA I/s, S. I80, Z. 20; LM, Bd. II, S. I 83 ; MEW, Bd. 3, S. 18 I (hinter ,klingen”).

/28/ [als wäre nun Jeder ein Eigen]thümer, [und d]aß die Bourgeois [...] der $\mathrm{He}[\mathrm{rr}]$ schaft der Eigenthümer [...] ausdr[...] dass jetzt die $s[\ldots]$ enthümer $[\ldots]$ die Bourgeoise par excel[lence ge]worden sei ${ }^{1}$. [...] „Schon am 8. Juli zer[störte die] Erklärung des Bischofs von [Autun] und Barrères den Schein, als [sei Je]der, der Einzelne, von Bedeu[tu]ng in der Gesetzgebung; sie [ze]igte die völlige Machtlosigkeit [de]r Committenten; die Majorität der Repräsentanten ist Herrin geworden." 2 - Die „Erklärung des Bischofs von Autun und Barrères" ist eine Motion, die Ersterer am 4. (nicht am 8.) Juli machte, und mit der Barrère Nichts zu thun hatte, als daß er sie am 8 . Juli unter vielen andern unterstützte. Sie ging am 9. Juli durch, wonach also gar nicht abzusehen ist, weshalb Sankt Max vom „8. Juli” spricht.

is Diese Motion „zerstörte" keineswegs „den Schein als sei Jeder, der Einzelne von Bedeutung" etc. - sondern sie zerstörte die bindende Kraft der den Deputirten übergebenen Cahiers, d.h. den Einfluß und die "Bedeutung", nicht von "Jedem, dem Einzelnen” sondern der feudalistischen 177 bailliages und 431 divisions des ordres; die Versammlung streifte durch Annahme dieser Motion den Charakter der alten, feudalistischen états generaux ab. Es handelte sich übrigens zu jener Zeit keineswegs um die richtige Theorie der Volksvertretung, sondern um höchst [P]raktische, unumgängliche Fragen. [D]ie Armee Broglies hielt Paris [i]m Schach und rückte täglich näher; die Hauptstadt war in der größten Aufregung; noch waren kaum I 4 Tage nach dem jeu-de-paume und dem lit-de-justice verflossen; der Hof intriguirte mit der Masse des Adels und der Geistlichkeit gegen die Nationalversammlung; endlich herrschte wegen der noch existirenden feudalistischen Provinzialdouanen u. als Folge der ganzen feudalen Agriculturwirthschaft eine Hungersnoth in den meisten Provin/28[a]./[zen,] und ein großer Geldmangel [mac]hte sich fühlbar. Es handelte [sich] in diesem Augenblick, wie Talleyrand selbst sagte, um eine assemblée essentiellement active, während die cahiers [der] adligen und sonstigen Reaktion dem Hofe Gelegenheit gaben, [den] Beschluß der Versammlung dur[ch Be]zug auf die Kommittenten f[ür nich]tig zu erklären. Durch die Talleyrandsche Motion erklärte sich die Versammlung für unabhängig, usurpirte die Gewalt, deren sie bedurfte, was natürlich auf politischem Boden nur innerhalb politischer Form und mit Benutzung der vorliegenden Rousseauschen

${ }^{1}$ Das Papier ist beschädigt,

${ }^{2}$ Stirner, a.a.O., S. 132 f. Vgl. Edgar Bauer, Bailly und die ersten Tage der Französischen Revolution (1843). 2. Aufl. Leipzig I847, S. 89 ff. 
etc. Theorieen geschehen konnte. (vergl. Le point du jour, par Barrère de Vieuzac I 789 , No. Is u. I7), Die Nationalversammlung mußte diesen Schritt thun, weil sie von der zahllosen Masse, die hinter ihr stand, vorwärts gedrängt wurde. Sie machte sich dadurch also keineswegs zu ,einer vollkommen egoistischen Kammer, abgelöst von aller Nabelschnur und rücksichtslos", sondern sie machte sich dadurch erst zum wirklichen Organ der großen Masse der Franzosen, von der sie sonst zertreten worden wäre, wie dies späteren „von aller Nabelschnur sich ablösenden" „vollkommen egoistischen" Deputirten auch geschah ${ }^{1}$. Sankt Max aber sieht vermittelst seines Io Geschichtsmäklers hier nur die Entscheidung einer theoretischen Frage; er sieht die Constituante, sechs Tage vor dem Sturm der Bastille, für ein Konzil von Kirchenvätern an, die sich ${ }^{2}$ über einen dogmatischen Locus disputiren! Übrigens ist die Frage über „die Bedeutung Jedes, des Einzelnen" nur in einer demokratisch ge- is wählten Repräsentation möglich, und kam während der Revolution erst im Konvent zur Sprache, aus ebenso empirischen Gründen, wie hier die Frage der Cahiers. Was die Assemblée constituante auch theoretisch entschied, war der Unterschied der Repräsentation einer herrschenden Klasse von der Repräsentation der herrschenden 20 Stände, und diese politische Herrschaft der Bourgeoisklasse war durch die Lage jedes Einzelnen, weil durch die damaligen Produktionsverhältnisse bedingt. Die Repräsentation ist ein ganz specifisches Produkt der modernen bürgerlichen Gesellschaft, d[as] ebensowenig von ihr zu trennen ist wi[e das] moderne einzelne Individuum.

Wie Sankt Max hier die 177 bailliages und 43 I divisions des ordres für „Einzelne" hält, so sieht er nachher im absoluten Monarchen und seinem car tel est notre plaisir die Herrschaft "des Einzelnen” gegenüber dem constitutionellen Monarchen, der "Herrschaft des Spuks (p I4I) ${ }^{3}$ und im Adligen, im Zunftmitgliede das wiederum „Einzelne” im Gegensatz zum Staatsbürger ( $p$ I 37 ).

„Die Revolution war nicht gegen das Bestebende gerichtet, sondern gegen dieses Bestehende, gegen diesen bestimmten Bestand." p. $145^{4}$. Also nicht gegen die bestehende Organisation des Grundeigenthums, der Steuern, der den Verkehr an allen Ecken hemmenden Douanen, und die

\footnotetext{
1 Stirner, a.a.O., S. 147.

2 Versehentlich nicht ausgestrichen von der alten Konstruktion: sich... berathen!

3 Vg]. ebd. S. 163.

"Bei Stirner (a.a.O.): „... sondern gegen dieses Bestehende, gegen einen bestimmten Bestand.”
} 


\section{ANHANG: TEXTVARIANTEN}

In allen Fällen, wo im Haupttcxt oder bei den ausgestrichenen Teilen des Textes Marxens Handschrift vorliegt, wird dies, falls es nicht bereits in Fußnoten vermerkt ist, durch ein ${ }^{m}$, die nachträglich von Engels in den von ihm geschriebenen Text eingeschalteten Stellen werden durch ein e gekennzeichnet., Der Beginn und Schluss nichthorizontaler Tilgungen wird durch $\mathbf{x}_{\ldots} . . \times$ horizontale Streichungen werden durch $\langle$ angedeutet. [] bezeichnen vermutliche Ergänzungen im Manuskript nicht ausgeschriebener Wörter. *bezeichnet entweder größeren Abstand zwischen Teilen der Randbemerkungen im Text oder den Beginn nachträglicher Ergänzungen zu Worten, auf die allein sich dann die Verfasserangabe $(\mathrm{m})$ bezieht.

In der linken Spalte neben dem Text auf S. 96, Z. 3/34 und (ab/) auf der Rückseite (vgl. MEGA I/s, S. 84 , Z. I 7 - S. 84 , Z. 2 I, 26/27, u. S. 8 , Z. II - S. 86, Z. 29, sowie S. 575 unten).

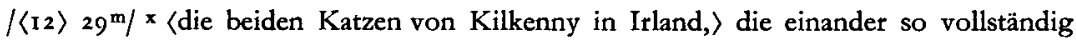
auffraßen daß zuletzt nur die Schwänze übrig 〈blieben〉. Ueber diese Schwänze spricht nun (der heilige) St. $m$ Bruno das Urtheil aus, daß sie „Substanz”, also auf (ewig verdammt sind. Hierbei〉 Schließliche beruhigt sich Herr Bruno mit dem Gedanken, daß ihm dem $^{e}$ Kritiker keine Kritik etwas anhaben könne, $\langle\mathrm{da}\rangle$ weilm „er $\langle$ "〉 der Kritiker selber" sei. $\left(p^{\mathrm{m}_{1}}{ }^{24}\right)^{\mathrm{m} 1}$

Nachdem nun der heilige Mann auf (diese) angegebenem Weise mit Stirner und Feuerbach ${ }^{2}$ fertig geworden ist, nachdem er ferner dem „Einzigen” „jeden Fortschritt abgeschnitten"m hat, wendet er sich 〈jetzt〉 gegen die angeblichen Konsequenzen Feuer〈bachs, die deutschen Kommunisten〉. Der heilige Vater mußte natürlich eine Gelegenheit, wie die vorliegende, abwarten, um sich den Kommunismus und seine theoretischen Vertreter in Deutschland nach Bedürfniß zurechtmachen unde damite beseitigene zu

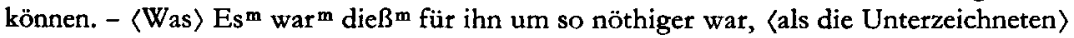

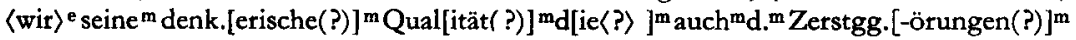
in der „heiligen Familie" $\mathrm{zu}^{\mathrm{m}}$ Protocoll ${ }^{\mathrm{m}}$ gebracht ${ }^{\mathrm{m}}$ 〈den Kommunismus speziell der „,kritischen Kritik" 〈ge[genüber]〉 als 〈letz[te]〉 äußersten Spitze der deutschent Theorie gegenüber geltend gemacht hatten $\rangle$. Der erste Eindruck den die „heilige Familie” auf den ehrwürdigen Kirchenvater macht, ist der einer tiefen Betrübniß und einer ernsten, bieder(männischen Wehmuth.) Die einzige gute Seite des Buches - daß es zeigte, ,was Feuerbach werden mußte und wie sich seine Philosophie stellen kann wenn sie gegen die Kritik kämpfen $\langle w i l l\rangle-{ }^{\prime m}\left(\mathrm{p}^{\mathrm{m}}{ }^{\mathrm{I}} 3^{8}\right)^{\mathrm{m}} \mathrm{daß}$ es also auf eine ungezwungene Weise das Müssen mit dem Können und 〈Wollen vereinigte,〉 - wiegt dennoch die vielen betrübenden Seiten nicht auf. 〈Die Feu[erbachsche]〉. Der Jammer des heiligen Bruno 〈ist〉 war darüber ist gleicht dem Jammer des alten Jehova, der durch alle vier großen und zwölf kleinen Propheten hindurch hinter ${ }^{e}$ seinem heiligen abtrünnigen ${ }^{e}$ Volk Israel noch herlamentirt, damit es ihn nur ja nicht ganz verläßt.) Die Feuerbachsche, hier 〈ganz willkührlich vorausgesetzte, Philosophie,> "darf und kann den Kritiker nicht verstehen - sie darf und kann die Kritik in ihrer Entwicklung nicht kennen und erkennen - sie darf und kann nicht wissen daß die Kritik / aller Transcendenz gegenüber ein immerwährendes Kämpfen und Siegen, ein fortdauerndes Vernichten und Schaffen, das einzig Schöpferische und Produzirende ist ... Sie darf und kann nicht wissen, wie der Kritiker gearbeitet hat und noch arbeitet, (um die transcendenten Mächte,) die bisher die Menschheit niederhielten

1 Hier folgt ein Trennungsstrich. - Vgl. S. 96, Anm. 3.

"Durch einen Strich Umstellung in „Feuerbach und Stirner" angedeutet.

1 Darüber: 〈sie seien nur ird[ische]Mühen (?) $^{m}$ 
und nicht zum Athmen und zum Leben kommen ließen, als das zu setzen und zu dem zu machen (!), was sie wirklich sind, als Geist vom Geist, als Inneres aus dem Inneren, als heimathliches (!) aus und in der Heimath, als Produkte (G[eschöpfe] Geschöpfe des Selbstbewusstseins〉. Sie darf und kann nicht wissen wie einzig und allein der Kritiker die Religion in ihrer Totalität, den Staat in seinen verschiedenen Erscheinungen gebrochen 〈hat, weil etc. etc. etc. " " p. ${ }^{\mathrm{m}}{ }_{13} 8^{\mathrm{m}} 139^{\mathrm{m}}$. Ist es nicht auf ein Haar der alte Jehova, der seinem durchgebrannten Volk, das an den lustigen Göttern der Heiden mehr Spaß findet, nachläuft und schreit: Höre mich Israel, und verschließe dein Ohr nicht, Juda! Bin ich nicht der Herr dein Gott der dich aus Egyptenland geführet hat in das Land da Milch und Honig fleußt - und siehe ihr habt von 〈Jugend auf gethan das mir übel ge)fällt, und habet mich erzürnet durch meiner Hände Werk, und habt mir den Rücken und nicht das Angesicht 〈zugekehret〉, wiewohl ich sie stets lehren ließ, und habet mir ihre Greucl in 〈das〉 meine Haus gesetzt, daß sie es verunreinigten, und haben 〈ihre〉 die Höhen des Baals (Feuerbach?) gebaut im Thale Ben Himmon, davon ich ihnen nichts befohlen habe und ist mir nicht in den Sinn gekommen daß sie solche Greuel thun sollten; und habe zu euch gesandt meinen Knecht Jeremiam, zu dem mein Wort geschehen ist von dem dreizehnten Jahr des Königes Josia, des Sohnes Amons, bis auf diesen Tag, und derselbige hat euch 〈gepredigt〉 nun dreiundzwanzig Jahr mit Fleiß gepredigt, aber ihr habt nie hören wollen 〈,〉. Darum (spricht der Herr Herr〉: wer hat je 〈so greuliches Ding) dergleichene gehört, 〈als〉 daß die Jungfrau Israel so gar greuliches Ding thut? Denn das Regenwasser ${ }^{\text {x }}$

Zu I), S. 96 (bis Z. 34 in der rechten Spalte neben dem oben abgedruckten Text, ab Z. 35 auf der Seite mit den Marx-Zeichnungen).

4 der 〈angefu[ehrten] $\rangle$ erwähnten 8 spricht 〈daher〉 Feuerbach

in die Natur, die 〈deren〉 noch nicht is das Wasser, 〈da um 20-2I Dampfschiffe befahren 〈wird〉, sobald sein Wasser in Gräben geleitet wird (die man ableiten) in denen 25-27 daß nämlich 〈die Abnormität und〉 dieser Widerspruch ihr eigner Widerspruch diese ${ }^{e}$ schlechte Lage $^{e}$ ihre $^{e}$ eigne $^{e}$ schelchte $^{e}$ Lagee se[i] $]^{\mathrm{e}}$ sei, wobei verschieden

$35 / \mathrm{r} / \times$ mühe aber und abermal 〈nach[gewiesen]〉 widerlegt worden ist, daß die heiligen Philosophen und Theologen die "Unselbstständigkeit des Individuums” „hervorgebracht hätten, $\langle\mathrm{da}\rangle$, indem sie" - einige abgeschmackte Phrasen über den absoluten Geist machten. Als ob das „Individuum” d.h. jeder Mensçh dadurch „unselbstständig würde, dadurch wirklich ,in den absoluten Geist〈"〉 aufgehoben"würde, wenn einige spekulirende Gedankenkrämer, die selbst erst 〈durch〉 - nicht durch die „(Abhängigkeit〉 Unselbstständigkeit e des Individuums" sondern durch einen lumpigen sozialen Zustand auf ihre philosophischen Marotten kommen konnten, dies dem „Individuum vorschwatzten, ihm beföhlen, es solle augenblicklich und ohne Raisonniren ,in den absoluten Geist aufgehen"! $\times$ Wir werden uns

1 Von hier an Fortsetzung des nicht getilgten Textes (S g6, Z. 35). - Wie bereits erwähnt,
hat Marx die ganze rechte Spalte dieser Seite mit Zeichnungen ausgefüllt. Neben dem
getilgten Text in der rechten Spalte außerdem Schreibübungen und z.T. kaum lesbare
Notizen von Marx (vgl. Bildtafel), die eine Einheit bilden, obwohl die letzten "Zeilen"
unter dem Trennungsstrich stehen. Die nicht mit Sicherheit zu entziffernden Teile der
Randbemerkungen wurden mit [], Ergänzungen mit () kenntlich gemacht: Schließlich 
Zu S. 97.

I aufzuklären, daß 〈damit〉 die „Befreiung” wenn sie 〈R[eligion]〉 Philosophie $6 \mathrm{da} \beta$ (man) es in 〈hinreichender〉 vollständiger Qualität 〈vorgef[aßten]〉 verschiednen 2I Natürlich 〈nehmen〉 ersetzen ${ }^{e}$ in 〈diese[m]) einem Lande

2-3 gekommen ist, $\langle\mathrm{da}[\mathrm{B}]\rangle$ S er nie 〈geseuf $[z t]\rangle$ geknechtet war 7 in $\operatorname{der}^{\mathrm{e}}$ wirklichen Welt $\quad I 2$ Kleidung $I 7$ nachträglich, 〈den〉 je nach ihren Ig Kritik 〈und beseitigen ihn wicder) gerade 23-24 Gedankenentwicklunwo 〈keine〉 nur ${ }^{e}$ cine $^{e}$ lumpige ${ }^{e}$ geschichtliche gen, diese ${ }^{e}$ verklärten ${ }^{e}$ und ${ }^{2}$ thatlosene Lumpereiene den 〈ie Stelle〉 der geschichtlichen 25 ein Kampf 〈der nur〉 von ${ }^{m}$ lokaler ${ }^{m}$ 〈Bedeu[tung] keine geschichtliche, allgemeine〉 Bedeutung 〈hat, ein Kampf der ebensowenig der〈ie Men[schen]〉 Masse der Menschen neue〈Tha[ten]〉 Resultate bringt, wie der Kampf der Civilisation gegen die Barbarei 〈, der Kampf des A[lten] ${ }^{1}$

x Der heilige Bruno gibt 〈also〉 eine "Charakteristik Ludwig Feuerbachs" d.h. eine verbessserte Auflage〈des〉 eines bereits in den „Norddeutschen Blättern” abgedruckten Aufsatzes $\langle,\rangle^{\mathrm{m}}\langle$ Bei dieser heilige Charakter worin in beiden wir und $\rangle$ Feuerbach wird ${ }^{\mathrm{m}}$

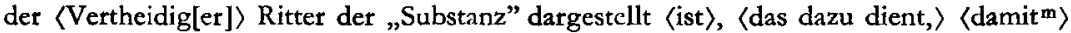

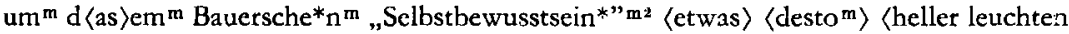
lassen〉. Bei dieser 〈Ver[wandlungl〉 Transsubstantiation Feuerbachs hüpft der heilige Mann 〈indeß〉 von (Ba[yles]) F[euerbach]'s Schriften über Leibnitz und Bayle sogleich auf das „Wesen des Christenthums herüber $\left\langle u^{m}{ }^{m}\right.$ überspringt ${ }^{m}$ 〉 〈ohne Feuerbachs den ${ }^{m}$ Aufsatz gegen die "positive" Philosophie $\rangle\langle z u\rangle\langle$ in den deutschen $\rangle$ hallischen ${ }^{m}$ 〈Jahrbüchern zu erwähnen ${ }^{4}$ aus dem einfachen Grunde weil hier〉 Feuerbach, enthüllte ${ }^{m}$ hier $^{\mathrm{m}}$ nähmlichm den positiven Vertretern der „Substanz" gegenüber, die ganze Weisheit vom 〈,absoluten〉,"m Selbstbewußtsein" 〈ver[urteilte(?)]〉 zu einer Zeit 〈aussprach $\rangle$, wo der heilige Bruno noch über die unbefleckte Empfängniss [spekulir]te.-m 〈und viel klarer, viel $[\ldots]$ aussprach, als $\operatorname{der}[\ldots]$ dies je gethan $[\ldots]\rangle^{\mathbf{x}^{5}}$

* Philosophische Schl(äge?) außtheilen * [Feuerbach wie scine] übrigen Concurrenten, glaubt d. Philosophie überwunden zu h(a)b(en). Der Kampf gegen die Allgemeinheit, den bisher das Individuum [geführt] hat, resumirt d. [Bestrebungen d.] deutschen phil. Kritik. Wir behaupten, daß dieser $K(\mathrm{am}) \mathrm{pf}$ in der Weise, wie es [ausgeführt] wird (oder: wurde), [beruht] selbst auf philosophischen Illusionen. [Wir begreifen,] daß die Allgemeinheit [unerteicht] war.

${ }_{1}$ Hiernach Beginn des S. 97, Anm. Io erwähnten Absatzes, neben dessen Beginn Marx in der rechten Spalte schrieb: Feuerbach. Bauer. of. Brumo über Feverbach, als d. Ritter d. Substanz.

${ }^{2}$ Hier von Marx in der rechten Spalte: x größeren Relief zu 〈geben〉〈verleihn〉. Ueberhaupt beschränkt sich (seine) die reine (Kritik) neuerdings darauf von Allem u. Allem zu sagen, daß es d. „Substanz” sei. ${ }^{x}$

'Ursprüngl. Reihenfolge: Bayle und Leibnitz.

"Hier von Marx in der rechten Spalte: Dieses 〈war Nachlässigkeit〉 „Versehn” war 〈nothwendig, weil〉, an der Stelle", 〈weil〉

- Hier von Marx in der rechten Spalte: xGrade in diesem Aufsatz untersucht Feuerb. das d. St. Bruno noch immer beschäftigende [über]haupt als d. [...].* - Das Papier ist beschädigt, daher Textverlust. - Vgl. E. Barnikol, Das entdeckte Christentum im Vormärz, Jena 1927, S. 31 , Anm. I. 
Zu II), S. 99.

3-4 die eBourgeoise1 $^{e 1}$...] 〈di[e... Bou]rgeois >herrschaft ersetzt durch dere Herrschaft dere Eigenthümere $1 \quad s$ [Eig]enthümer [...s] $\langle$ ei; was ihnen [...] schon läng[st] vor der Revo[lution $z]$ um Vorwurf gemacht worden $\rangle[\ldots]$ die Bourgeoise

die "mBedeutung"m $m$ I 9 der feudalistischen ${ }^{\mathrm{m}} 77$ bailliages und 431 division*s $\mathrm{m}$ $2 I$ états generaux 〈wirklich〉ab 23 unumgängliche 〈nöthi[ge]〉

Fragen 29 Provinzialdouanen ((cantonnement)) von Marx in der rechten Spalte durch folgende Bleistiftnotiz ersetzt: u. als Folge der ganzen 〈W[irtschaft]〉 feudalen Agriculturwirthschaft $\quad 31 \quad 28[\mathrm{a}] .^{\mathrm{m}} \quad 33$ essentiellement active, 〈und die cahiers, die fast ${ }^{\mathbf{e}}$ Alle auf Beibehaltung der Zunft- und Adelsprivilegien drangen, hätten die Thätigkeit der Versammlung vernichtet.) in der recbten Spalte von Engels ersetzt durcb: während die 〈Gült[igke]it〉 cahiers bis zu erklären.

(geschehen konnt[e]) und mit Benutzung

S. 100 .

I-Io von (vergl. Le point bis geschah von Engels in der recbten Spalte binzugefingt 2

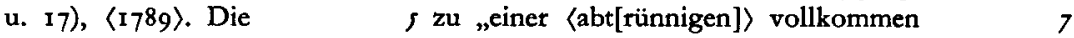

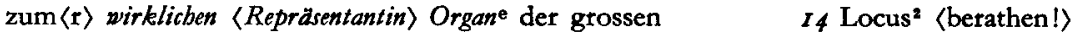
disputiren! I6 Repräsentation (überhaupt) möglich 18 Cahiers. 〈Da Stirner hiervon nicht spricht, brauchen wir auf diesen Punkt auch nicht einzugehen〉. in der rechten Spalte von Engels ersetzt durch: Was die Assemblée bis Individuum Ig war $\mathrm{d}\langle\mathrm{ie}\rangle$ er Unterschied $2 I$ diese politische ${ }^{e}$ Herrschaft

23 ein ganz〈modernes〉 specifischese Produkt 2f 〈das〉 moderne einzelne Individuum 33 gegen diesen $\langle\mathrm{Be}[\mathrm{stand}]\rangle$ bestimmten Bestand

1 Von Engels in der rechten Spalte ergänzt.

${ }^{2}$ Das c von Marx mit Bleistift verdeutlicht. 